\title{
Analysis of High School Students' Academic Stress During the Covid-19 Epidemic
}

\author{
${ }^{a}$ Nanjing Foreign Language School, Nanjing, 210008, China, \\ ${ }^{b}$ Bellerbys College Brighton, Brighton, UK, BN1 4LF, \\ ruilin tian@126.com \\ b2760317953@qq.com
}

Ruilin Tian ${ }^{1, a}$, Jiayuan $\mathrm{Li}^{1, \mathrm{~b}}$

\begin{abstract}
This essay mainly looked at the stress among high school students during pandemics and the internal and external causes of which. This study was conducted because depression and anxiety were wildly found among students especially during the covid pandemic and the author hopes to call attention to these negative emotions existing in the specific group. By reviewing essays on relevant topics, it can be found out these factors mainly include parental distress, lack of in-person communication and sedentary lifestyle. Nevertheless, all the research tends to focus more on the students themselves and further research could look more into what schools and families could do in order to resolve the problem.
\end{abstract}

Keywords: Students, Stress, Covid-19, Problems

\section{INTRODUCTION}

At the end of 2019, the first cases of a novel coronavirus were reported in Wuhan, China, and were later linked to the respiratory disease known as Covid-19. On 11th March 2020, due to the rapid prevalence of Covid-19, World Health Organisation (WHO) declared it a global pandemic [1]. Several critical restrictions, such as banning public gatherings, reducing commuting to the workplace, and closing universities and schools, were taken by most countries to take control of the spread of the pandemic. However, the closure of schools forced students to study at home. Negative psychological symptoms such as boredom, followed by loneliness and frustration are most frequently reported of children by their caregivers in the UK during lockdown [1]. About one-third of caregivers also reported emotional problems such as irritability, irritability, anger, anxiety, sadness, worry, and being prone to argue with other family members [1]. Among university students in French, the average PSS score was relatively high, and many of them have experienced perceived stress [2]. In China, almost half of adolescents experienced psychological distress (e.g., post-traumatic stress disorder [14\%], negative coping, stress) after COVID-19 was announced as a public health emergency [3].

Importantly, according to Zhang et al, high school students show more symptoms of anxiety and stress than junior high school students do [4]. Being high school students ourselves, the author also has experienced pressure during the lockdown period. Therefore, the pressure for high school students (Year 10 and on) during the lockdown period has caught the author's attention. It is clear that Covid lockdown and restrictions have brought about detrimental effects to students' psychological status and have threatened their mental health at a crucial stage of growth. Thus, it is essential for the public to realise the issue and take measures to address it immediately. For this purpose, this study will identify several emotional problems related to stress,as well as the situational and personality factors that cause high school students to be stressed. Aiming at these results, the author will discuss and propose some constructive solutions to attract more public attention to this issue and improve students' mental health during the pandemic.

\section{MAIN BODY}

\subsection{Students'situation during the COVID-19}

During the lockdown in the period of COVID-19, high school students' situation became much worse than before the pandemic. They inevitably faced a lot of difficult situations, which caused many mental problems. 


\subsubsection{Ineffective online study}

The first situation was about the field of online study. In order to stop the spread of the COVID-19 to campuses and ensure the safety and health of teachers and students, the Ministry of Education issued a notice asking students to stay at home and not to go out or participate in centralized activities. Schools then canceled offline classes and replaced them with online classes as required. Online classes only required students to sit in front of their computers, enter virtual classrooms, and listen to the teachers. Students could also control the on and off of their cameras and microphones. Therefore, the monitoring of students' learning status was relaxed to a certain extent, and some students with poor self-control ability, Will not be able to concetrate on their class. With such a high level of freedom, they might play games, watch videos, and chat with friends during online classes. These behaviors significantly affected learning efficiency because they would miss a lot of knowledge and could not absorb knowledge effectively. Research conducted by researchers in Anhui, China showed that $76.95 \%$ of students thought that the COVID-19 had an adverse impact on their studies and that online classes are ineffective. [5] Another online research shows that 56.9\% of students believe that the efficiency of online classes at home is lower than that of classes at school, and $54.8 \%$ of students spent less than eight hours effectively studying at home during the epidemic. [6] As a result, many students did poorly in the exams, which caused serious psychological stress on them.

\subsubsection{Bad Study Status}

The second situation was also about the study. Social activities have a huge effect on students' study status. Normally, during the break and after school, students like to chat with each other, play games, and do some sports, which can all help them to relax and regulate themselves. However, with the implementation of the Home Isolation Policy, students could no longer interact with friends and their relationship with classmates became worse. It has been reported that the percentage of high school students with mild interpersonal tension increased from $15.0 \%$ to $18.2 \%$ and the percentage of high school students with severe interpersonal tension increased from 0 to $0.5 \%$.[7] What's more, students' pressure gained during the classes could not be released. As a result, they could not maintain good study status for a long time and their study motivation decreased. All these things resulted in increased pressure on their minds. A study in Shandong found that $49.9 \%$ of the students had different degrees of depressive symptoms and $31.6 \%$ of students had different degrees of anxiety symptoms. [6]

\subsubsection{Feeling of Loneliness}

The third difficult situation students faced was the feeling of loneliness. As mentioned above, the implementation of the Home Isolation Policy forced the students to stay at home during the pandemic, and they could seldom have interaction with the outside world. This caused a huge psychological gap in their mind and made them feel lonely. Research showed that $89.27 \%$ of students experienced mild to severe levels of loneliness during the quarantine. [8] This situation was even worse for some international students. They already suffered the isolation between their parents and themselves before the spread of COVID-19, and had to rely on people around to weaken the feeling of loneliness. During the pandemic, they lost even this last way and their loneliness was more serious than ever.

\subsection{Emotional problems related to stress}

In China, compared with junior high school students, high school students score higher in the Depression, Anxiety and Stress Scale (DASS) and suffer more from the Covid lockdown (16.28 for junior high school students and 19.77 for high school students) [4]. Also, a similar rate of depression and trauma-related distress symptoms are shown in junior high and high school students, and a higher rate of anxiety and stress is shown in the latter [4]. Nevertheless, for both high school and junior high school students in China, the proportion of depression symptoms and anxiety symptoms were raised compared with the proportion reported by previous studies that did not include Covid lockdown or any pandemic [4]. In Greece, these rates showed a similar pattern (depression rate increased from $10 \%$ before pandemic to $27 \%$ during a pandemic; anxiety rate from $3.85 \%$ to $20.5 \%$ ) [9]. Apart from depression and anxiety, children in the UK showed more symptoms of being bored $(73.8 \%)$, lonely $(64.5 \%)$, sad $(43.4 \%)$, frustrated $(61.4 \%)$, irritable $(57.1 \%)$, restless $(52.9 \%)$, worried $(52.4 \%)$, angry $(48.6 \%)$, and were more likely to argue with the rest of the family $(29.7 \%)$ during the lockdown compared to the pre-COVID-19 period.

\subsection{Factors causing the problems}

\subsubsection{Parental distress}

In Morgül's study, children being more worried, restless, anxious, sad, lonely, uneasy, nervous, angry, frustrated, bored, and irritable were most reported by those caregivers who themselves with a higher level of distress during the lockdown. Despite the possibility that these distressed caregivers were perceiving their children more negatively with bias, the correlational study by Leijdesdorff et al shows that the association between poor parental mental health and poor children outcomes is strongly related. This may suggest that it is the caregivers' 
emotional problems causing those of their children during the lockdown. Though not as crucial as it is for infants, parents' attention towards children's emotions is important to teenagers as well. According to Pinquart, the obstacle reacting to a child's emotional need experienced by some parents with mental illness could trigger aggressiveness and negative behaviours in children. Especially high school students in adolescence that generate an enormous amount of hormone will be more susceptible to bad emotions such as anger and sadness. With the lack of parental care and response during the lockdown, children at this age could be more vulnerable to emotional problems like anxiety and depression.

\subsubsection{Lack of in-person communication}

It was found that $65 \%$ of students in Britain wanted to return to school for socialisation reasons [10]. Before the pandemic, the school worked as an institute for children to cultivate their social abilities such as participation, self-confidence and empathy. However, during the lockdown, children lost a physical space to share their common interests, thoughts, feelings, as well as expectations with their peers. Indeed, social media and online communication tools such as Skype and Zoom do allow students to maintain essential communication with teachers and classmates and satisfy their basic social needs, yet they are not as effective as in-person communication in terms of transporting emotions and empathy, therefore unlikely can they become the substitute of real-life interaction. Although there is no study showing the positive correlation between stress and the absence of face-to-face interaction, being in a crucial stage of developing their values and interests, it is reasonable to take the missing of in-person communication into account considering factors causing stress and loneliness among high school students.

\subsubsection{Sedentary lifestyle}

The lockdown measures against Covid-19 increase the time people spend at home, which has led to an increase in the time spent on electronic devices and a reduction in time spent on physical activity. A survey among parents in Italy and Spain shows that more than $80 \%$ of their children use the electronic device more than 1 hour per day, and only $14.8 \%$ practice physical activities for more than an hour, suggesting that this may result in problems of anxiety, less sleep as well as health problems [11]. National data shows that Spanish children show more stress-related symptoms than Italian children do. The study suggests that this may be due to stricter confinement on physical activity in Spain, as well as less access to private gardens among Spanish families (30\%) percent less. Studies also have shown that aerobic exercise can work as an antidepressant and anxiolytic reducing stress-related symptoms [12]. Therefore, without the regular essential physical activities conferring resilience against stressors, students are more likely to be affected by negative emotions.

\section{DISCUSSION}

There are several ways to relieve students' stress and solve their mental problems.

\subsection{Loving-kindness Meditation}

The first way is to use the Loving-kindness Meditation. The core of Loving-kindness Meditation is to pass on blessings to others. The first step is to sit in meditation with students' eyes closed and think of the 3 to 4 blessings they have for themselves. Then, think about someone they would like to thank, and convert the blessing to him and say it out loud. Next, think about someone they hate and pass the blessing to that person in the same way. Finally, think of someone they neither thank nor hate, and repeat the blessing they just gave.

Research shows that passing on blessings not only brings positive energy to people, but also increases their sense of the experience of positive emotions, resistance to depression, and satisfaction with life. [13] At the same time, the act of "passing on the blessing" itself creates a sense of social connection, making people feel more connected to others and reducing feelings of isolation.

\subsection{Daily Gratitude Journal}

Taking a daily gratitude journal is also a very useful way. It's a very easy way: choose a time of day when students have several minutes to step outside their life and thoughtfully reflect. Then write down at least five things for which they are currently grateful. Students can also take a photo of things they are thankful for too. Do this each day for the whole week.

Expressing people's gratitude can increase their overall happiness. It has been proved that grateful people have higher levels of agreeableness, openness, and lower neuroticism traits. Expressing gratitude can also make people think optimistically. Dr. Emmons and Dr. McCullough conducted a study in 2003 to examine the impact of gratitude. Their study showed that after 10 weeks of practice, those who focused on gratitude showed more optimism in many areas of their lives. When people are optimistic about their well-being and health, they are more likely to have healthy lifestyles.[14] What's more, people who always express gratitude have stronger self-control. A 2014 study led by De Steno found that self-control increased significantly when participants felt emotions of gratitude.[15] Self-control helps people to be more focused and can lead to long-term happiness. 


\section{CONCLUSION}

During the COVID-19, the governments introduced the Social Isolation Policy to control the spread of the epidemic. High school students, therefore, had to stay at home and take online classes. This caused a lot of difficult problems, including the low efficiency of online study, the lack of social interactions, and the feeling of loneliness, which all contribute to the huge stress and even mental illnesses. A large proportion of high school students show symptoms of depression, anxiety, loneliness, boredom, frustration, and irritability. These problems were mainly caused by poor parental mental health, lack of face-to-face interactions with others, and lack of physical activities. To reduce stress and solve mental problems, students can use the Loving-kindness Meditation and take a gratitude journal every day.

However, this research is not comprehensive enough. The author only discusses several ways students can do to relive stress and solve problems, but not people around them. Further research should focus on ways that parents and schools can do to help students conquer the difficulties.

\section{ACKNOWLEDGMENT}

Firstly, we would like to show our deepest gratitude to our phycology teacher Anthony Ong from Connell University, who gave us brilliant classes and taught us many important knowledges. We would also like to thank our essay teacher who provided us with valuable guidance in every stage of the writing of this essay. What' $\mathrm{s}$ more, we would like to pay respect to all the researchers that provide us with related papers. Those data and theories really help us a lot. Last but not least, we would like to thank all our friends and parents for their encouragement and support. Without all their enlightening instruction and impressive kindness, we could not have completed our essay.

\section{REFERENCES}

[1] Morgül, E., Kallitsoglou, A., \& Essau, C. A. E. (2020). Psychological effects of the COVID-19 lockdown on children and families in the UK. Revista de Psicología Clínica con Niños y Adolescentes, 7(3), 42-48.

[2] Bourion-Bédès, S., Tarquinio, C., Batt, M., Tarquinio, P., Lebreuilly, R., Sorsana, C., \& Baumann, C. (2021). Stress and associated factors among French university students under the COVID-19 lockdown: The results of the PIMS-CoV 19 study. Journal of affective disorders, 283, 108-114.

[3] Liang, L.; Ren, H.; Cao, R.; Hu, Y.; Qin, Z.; Li, C.; Mei, S. The Effect of COVID-19 on Youth Mental Health. Psychiatry Q. 2020.
[4] Zhang, C., Ye, M., Fu, Y., Yang, M., Luo, F., Yuan, J., \& Tao, Q. (2020). The psychological impact of the COVID-19 pandemic on teenagers in China. Journal of Adolescent Health, 67(6), 747755.

[5] Gong, T.; Yao, R. Investigation and countermeasure research on the psychological impact of novel coronavirus pneumonia epidemic on high school students[J]. Anhui Educational Research,2021(09):1-3.

[6] Yang, M.; Yang, C.; Zhang, Z.; Zhang, J.; Li, L.; Li, J.; Gao, S.; Zhou, C. A study on the mental health status of students in a high school in Shandong Province during a novel coronavirus pneumonia epidemic[J]. Campus Psychology,2020,18(06):517522.

[7] Wu, P.; Wang, Y. Mental health status and response of high school students under the normalization of new crown pneumonia epidemic prevention and control[J]. Strait Science,2021(5):95-97.

[8] Leodoro J. Labrague, Janet Alexis A. De los Santos, Charlie Falguera et al. Social and emotional loneliness among college students during the COVID-19 pandemic: the predictive role of coping behaviours, social support, and personal resilience, 19 October 2020, PREPRINT (Version 1) available at Research Square [https://doi.org/10.21203/rs.3.rs-93878/v1]

[9] Giannopoulou, I., Efstathiou, V., Triantafyllou, G., Korkoliakou, P., \& Douzenis, A. (2021). Adding stress to the stressed: Senior high school students' mental health amidst the COVID-19 nationwide lockdown in Greece. Psychiatry Research, 295, 113560 .

[10] Semple, F.; Mayne, D.E. Year 10 and 12 school students' opinions on returning to partial schooling during the COVID-19 pandemic: An action research prospective survey. Hilos Tensados 2020, 1, 1-476. [CrossRef]

[11] Orgilés, M.; Morales, A.; Delveccio, E.; Mazzeschi, C.; Espada, J. Immediate psychological effects of the COVID-19 quarantine in youth from Italy and Spain. PsyArXiv 2020.

[12] Salmon, P. (2001). Effects of physical exercise on anxiety, depression, and sensitivity to stress: a unifying theory. Clinical psychology review, 21(1), 33-61.

[13] Zeng, X., Chiu, C. P. K., Wang, R., Oei, T. P. S., \& Leung, F. Y. K. (2015). The effect of lovingkindness meditation on positive emotions: A meta- 
analytic review. Frontiers in Psychology, 6, Article 1693. https://doi.org/10.3389/fpsyg.2015.01693

[14] Emmons RA, McCullough ME. Counting blessings versus burdens: an experimental investigation of gratitude and subjective well-being in daily life. $\mathbf{J}$ Pers Soc Psychol. 2003 Feb;84(2):377-89. doi: 10.1037//0022-3514.84.2.377. PMID: 12585811.

[15] DeSteno D, Duong F, Lim D, Kates S. The Grateful Don't Cheat: Gratitude as a Fount of Virtue. Psychological Science. 2019;30(7):979-988. doi:10.1177/0956797619848351 